\title{
Flexible Optical Dipole Mirror for Cold Atoms
}

\author{
T. KawaleC ${ }^{*}$, K. Kiersnowski, J. Fiutowski \\ AND T. DOHNALIK \\ Smoluchowski Institute of Physics, Jagiellonian University \\ Reymonta 4, 30-059 Cracow, Poland
}

(Received May 25, 2008)

\begin{abstract}
A flexible and relatively simple and cheap optical dipole mirror for cold rubidium atoms from magneto-optical trap is described, being a very modern and efficient tool for atomic physicists. Emphasis is put on physical processes responsible for the mirror parameters and their optimization. Promising perspectives are provided for evanescent wave properties investigation and atom-surface interaction measurements.
\end{abstract}

PACS numbers: 37.10.De, 37.10.Vz

\section{Introduction}

Lasers provide the possibility of effective cooling and trapping of neutral atoms, allowing one for precise spectroscopic measurements, atom internal and external degrees of freedom manipulation and investigation of quantum effects. One of the methods of atom movement control bases on the interaction between an atom and an off-resonance, intense radiation with high intensity gradient, via so-called dipole force [1]. In particular, a good choice for such a radiation is an evanescent wave (see e.g. [2]), as pointed out in 1982 by Cook and Hill [3]. The evanescent wave may be created on the vacuum side of a vacuum-dielectric interface when the light undergoes total internal reflection. Providing the wave is blue detuned from a given optical transition in an atom, a repulsive force acts on it in the direction perpendicular to the dielectric surface. The elastic optical dipole mirror was realized for the first time in 1987 for thermal atoms [4] and in 1990 for cold atoms from a magneto-optical trap (MOT) [5]. In 1995 there was proposed [6] and realized [7] the inelastic optical mirror, in which under certain circumstances, efficient cooling of the atomic sample occurs during reflection thanks to a spontaneous Raman transition between ground hyperfine sublevels [8].

*corresponding author; e-mail: poczta@kawalec.biz 
Evanescent wave dipole mirror was used amongst other for the van der Waals force measurements between atoms and dielectric surface [9], and atom optics experiments [10-12]. It formed also a support for creation of a two-dimensional Bose-Einstein condensate [13].

Mirrors, traps, and guides for atoms have been also successfully implemented in surface micro-devices, using magnetic forces [14]. For some more recent advances in both optical and magnetic approaches see Ref. [15].

In this article a flexible, relatively cheap and simple evanescent wave optical dipole mirror for cold rubidium atoms is presented. Emphasis is put on mirror construction, performance and physical background description. Very promising perspectives are also provided, especially in the field of the Casimir-Polder interaction measurements, being an important issue in the domain of nanotechnology applications and basic research. The described optical dipole mirror may be also used to investigate evanescent wave properties.

Since the main aspects of the evanescent wave mirrors are presented in the reviews $[16,17]$ and in the articles (see e.g. $[18,8]$ ), only a basic introduction is given in the next section.

\section{Principle of operation}

The heart of the optical dipole mirror is a repulsive potential allowing elastic or inelastic reflections of atoms. In the optical surface mirrors, a blue detuned evanescent wave is used, providing large potential barrier because of its high intensity gradient in the direction perpendicular to the prism surface. For a Gaussian incident beam, the intensity of the evanescent wave reads

$$
I(\boldsymbol{r}, \theta)=I_{0}(\theta) \exp \left(-\frac{x^{2}}{w_{x}^{2}}\right) \exp \left(-\frac{y^{2}}{w_{y}^{2}}\right) \exp \left(-\frac{2 z}{d(\theta)}\right),
$$

where $\theta$ is an incidence angle of the beam forming evanescent wave, $I(\theta)$ is on-surface $(\boldsymbol{r}=0)$ maximal intensity, $d(\theta)$ is penetration depth of the evanescent wave, and $w_{x, y}$ are evanescent wave spot Gaussian $\left(\mathrm{e}^{-2}\right)$ radii. The angle $\theta$ is greater than so-called critical angle $\theta_{\mathrm{c}}[2]$. The directions $\hat{x}$ and $\hat{y}$ are in the prism surface plane and $\hat{z}$ direction is perpendicular to that plane (see also Fig. 2). The dipole potential $U_{F m}$ seen by the alkali atom in the ground state $|F, m\rangle$ for evanescent wave detuning large enough is as follows [18]:

$$
U_{F m}(\boldsymbol{r}, \theta)=\frac{3}{16} \frac{\Gamma \lambda_{0}^{3}}{\pi^{2} c} I(\boldsymbol{r}, \theta) u_{F m}, \text { where } u_{F m}=\frac{2 J^{\prime}+1}{2 J+1} \sum_{F^{\prime}} \frac{\left|C_{F m, F^{\prime} m}\right|^{2}}{\Delta_{F F^{\prime}}} .
$$

$\Gamma$ is a spontaneous decay rate, $\lambda_{0}$ is the wavelength of the evanescent wave, and $C_{F m, F^{\prime} m}$ are the Clebsch-Gordan coefficients for a given orbital momentum in the ground $(J)$ and excited state $\left(J^{\prime}\right) . \Delta_{F F^{\prime}}$ denotes the evanescent wave detuning in relation to $F \rightarrow F^{\prime}$ transition. It is often convenient to use the mean potential $U_{F}$, taken as an average value of $U_{F m}$ over magnetic sublevels $m$. The incoherent photon scattering by the atom in the evanescent wave field reads 


$$
\Gamma_{F m}(\boldsymbol{r}, \theta)=\frac{3}{16} \frac{\Gamma^{2} \lambda_{0}^{3}}{\pi^{2} \hbar c} I(\boldsymbol{r}, \theta) g_{F m}, \text { where } g_{F m}=\frac{2 J^{\prime}+1}{2 J+1} \sum_{F^{\prime}} \frac{\left|C_{F m, F^{\prime} m}\right|^{2}}{\Delta_{F F^{\prime}}^{2}} .
$$

In order to calculate the number $P_{F m}(\theta)$ of incoherent scattered photons per single atom reflection, one has to integrate Eq. (3) along the atom trajectory and gets

$$
P_{F m}(\theta)=\frac{m d(\theta) \Gamma}{\hbar} v_{z} \frac{g_{F m}}{u_{F m}},
$$

where $m$ is mass of an atom and $v_{z}$ is the $z$-component of velocity of an atom when reaching the mirror. The result shown in Eq. (4) extends the calculations presented in $[18,17]$ to the case of the detuning $\Delta_{F F^{\prime}}$ comparable with the excited state hyperfine splitting. Let us note that for pure evanescent field, $P_{F m}(\theta)$ does not depend on $I(\boldsymbol{r}, \theta)$. Incoherent photon scattering is very important for the dipole mirror performance, as explained later in Sect. 3 and for cooling processes, as described in Sect. 4. The total potential $U$ in the dipole mirror equals $U_{F}+U_{\mathrm{g}}+U_{\mathrm{vdW}}$, where $U_{\mathrm{g}}=m g z$ accounts for gravity and

$$
U_{\mathrm{vdW}}=-\frac{n^{2}-1}{n^{2}+1} \frac{28.2 e^{2} a_{0}^{2}}{48 \pi \epsilon_{0}} \frac{1}{z^{3}}=-\hbar \Gamma \frac{150 \times 10^{-6}}{z^{3}\left[\mu \mathrm{m}^{3}\right]}
$$

is the Lennard-Jones potential connected with the van der Waals force. Symbols $n, e, a_{0}$ and $\epsilon_{0}$ denote: prism refraction index, electron charge, Bohr radius, and vacuum dielectric constant, respectively [19].

The calculated potentials of the dipole mirror are shown in Fig. 1a for realistic experimental parameters. It is easy to see that the van der Waals force lowers significantly the barrier height for falling atoms. In Fig. 1b the numerically calculated atom trajectories are presented. The reflection time is of the order of several microseconds.
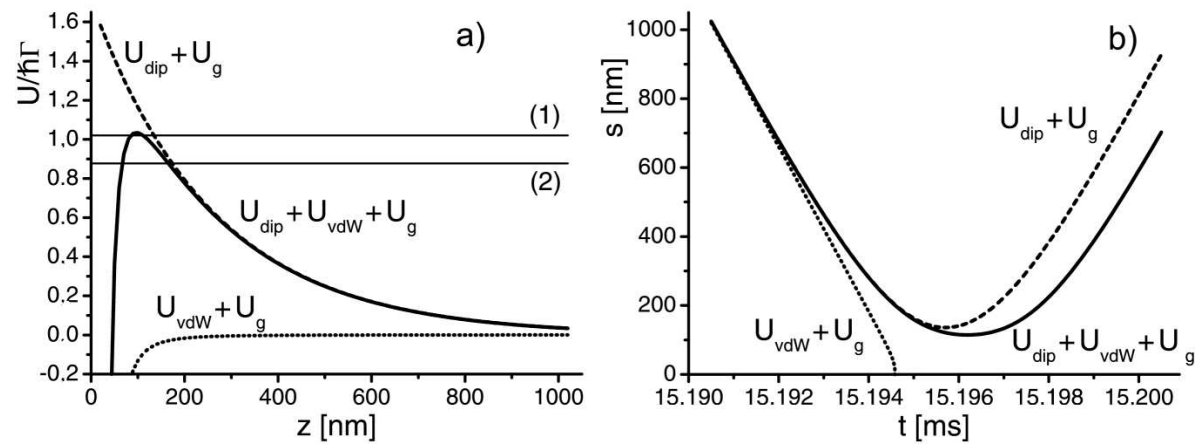

Fig. 1. (a) Calculated average potential in the dipole mirror for ${ }^{85} \mathrm{Rb}$ atom in the $F=2$ ground state and real experimental parameters: $I_{0}(\theta)=88 \times 10^{3} \mathrm{~W} / \mathrm{cm}^{2}, d(\theta)=520 \mathrm{~nm}$, $\theta=43^{\circ}=1.5^{\circ}+\theta_{\mathrm{c}}, \Delta_{23}=2 \pi 900 \mathrm{MHz}$ in relation to $5^{2} \mathrm{~S}_{1 / 2} F=2 \rightarrow 5^{2} P_{1 / 2} F^{\prime}=3$ transition. Thin horizontal lines denote the mechanical energy of the falling atom with initial thermal energy of $70 \mu \mathrm{K}(1)$ and at rest (2) for the initial height $h$ over the prism equal to $2.5 \mathrm{~mm}$, (b) calculated trajectory of the reflected atom for the case (1). 
The important parameter of the dipole mirror is its effective surface (see e.g. [9]), allowing for calculation and optimization of the bouncing atom fraction. Since the total potential of the dipole mirror changes in the $\hat{x}-\hat{y}$ plane (reflecting the profile of the incidence beam), it is possible to calculate the surface in the $\hat{x}-\hat{y}$ plane, for which the group of falling atoms with a given mechanical energy $E$ will be reflected from the optical mirror. The effective mirror radius $w_{\text {eff }}$ may be found by numerical solving the inequality $U_{F}\left(x, y, z_{\max }\right)+U_{\mathrm{vdW}} \geq E$ for $x$ and $y$ variables, where $z_{\max }$ is the distance from the surface, for which the total potential has its maximum.

\section{Experimental realization}

The scheme of the main part of the experimental setup is shown in Fig. 2a. The cold ${ }^{85} \mathrm{Rb}$ atom cloud is produced in a classical MOT setup. The rubidium source for our trap were two resistively heated alkali metal dispensers (Saes Getters). The number of atoms in the trap was roughly controlled by the dispensers' temperature, which was about $250^{\circ} \mathrm{C}$ for a typical current of $2.0 \mathrm{~A}$. The MOT was formed by the intersection of three orthogonal beams, retroreflected after passing the trap center. We applied diode lasers as a source of optical forces. The following $\mathrm{D}_{2}{ }^{85} \mathrm{Rb}$ lines were used as the cooling and repumping transitions respectively: $5^{2} S_{1 / 2} F=3 \rightarrow 5^{2} P_{3 / 2} F^{\prime}=4$ and $5^{2} S_{1 / 2} F=2 \rightarrow 5^{2} P_{3 / 2} F^{\prime}=3$. The cooling laser beam waist was $4.16 \mathrm{~mm}$, and the total power in all beams was 40 to $80 \mathrm{~mW}$. The gradient of the applied magnetic field was in the range of 16.0 to $18.5 \mathrm{Gs} / \mathrm{cm}$. The temperature of the atomic cloud, measured with a standard time-of-flight (TOF) technique, was $75 \pm 5 \mu \mathrm{K}$ for all measurements presented in this article.

The principle of operation of the MOT requires that the cooling radiation is red detuned from a closed optical transition up to several $\Gamma$ units. Usually this is achieved by stabilizing the radiation frequency to another transition and shifting the frequency by means of an acousto-optic modulator (AOM). We simplified this solution and we stabilized the beam frequency directly to one of the Zeeman components of the cooling transition in the saturated absorption spectrum. The frequency of this component was regulated by the magnitude of the homogeneous magnetic field applied to the reference $\mathrm{Rb}$ vapor cell. For the magnetic field induction in the range of $0 \div 22 \mathrm{Gs}$, the cooling beam was detuned by 0 to $-20 \mathrm{MHz}$ from resonance.

A glass (N-BK7) prism with the index of refraction $n=1.5109$ at $\lambda=795 \mathrm{~nm}$ is mounted inside the vacuum chamber, below the center of the MOT (see Fig. 2) so that the vertical pair of MOT beams is going through the prism. The prism may be resistively heated up to $500 \mathrm{~K}$ or cooled down with liquid nitrogen. The flatness is $\lambda / 20$ for the upper surface and $\lambda / 10$ for the other surfaces $(\lambda=800 \mathrm{~nm})$ and the roughness is $2 \mathrm{~nm}$ root mean square (RMS). The prism design allows for producing evanescent wave on the upper surface, however two total internal reflections are also used to guide the beam inside the prism (see 

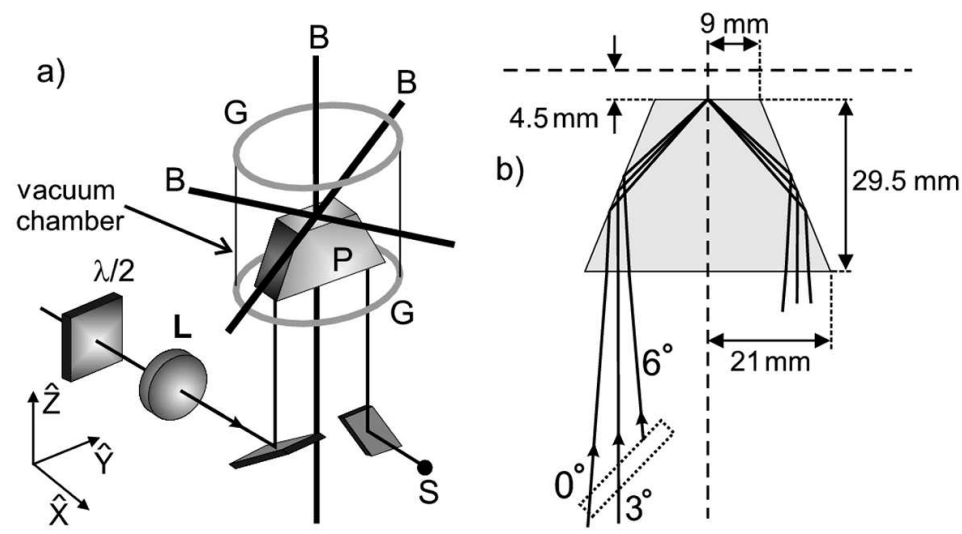

Fig. 2. (a) Sketch of the experimental setup: $\lambda / 2-$ half-waveplate, $L-150 \mathrm{~mm}$ converging lens, $P-$ glass prism, $S$ - saturation spectroscopy, $B-$ MOT beams, $G$ - magnetic gradient coils; (b) prism construction and mounting details (see text). The angles denote the difference between incidence angle $\theta$ and critical angle $\theta_{\mathrm{c}}=41.5^{\circ}$ on the upper prism surface. Prism thickness is $20 \mathrm{~mm}$. Horizontal thick dashed line represents the projection of the center of the MOT beams in the $\hat{x}-\hat{y}$ plane.

Fig. 2b). Further details are provided in Ref. [20]. The evanescent wave is created with EOSI 2001 single-mode diode laser with external Littman-Metcalf resonator. The beam power is $22 \mathrm{~mW}$ at maximum and the wavelength may vary between 793 and $802 \mathrm{~nm}$. In the experiments described here the laser was tuned to the blue wing $\left(200 \div 2000 \mathrm{MHz}\right.$ range) of $5^{2} S_{1 / 2} F=3 \rightarrow 5^{2} P_{1 / 2} F^{\prime}=3$ or $5^{2} S_{1 / 2}$ $F=2 \rightarrow 5^{2} P_{1 / 2} F^{\prime}=3$ transitions. The beam is shaped with $150 \mathrm{~mm}$ converging lens and its polarization is controlled by means of a half-waveplate. After passing the prism and the vacuum chamber, the beam is sent to a saturation spectroscopy setup for the purpose of the frequency determination.

The center of the MOT (i.e. the point of the minimum of the magnetic field) may be moved along vertical $(\hat{z})$ direction by means of asymmetric powering of the gradient coils. This method allows easily to change the initial height $h$ of the cold atom cloud above the prism surface. The $10 \%$ imbalance in gradient coils currents causes the cloud to be shifted by about $2.5 \mathrm{~mm}$. The prism and MOT beams configuration described above improves the performance of the dipole mirror in comparison to the other existing setups since the cold atom cloud can be created as close as $0.3 \mathrm{~mm}$ to the prism surface. The upper limit is $8 \mathrm{~mm}$ and is related to horizontal MOT beams diameter.

The most important parameter for the dipole mirror performance is the incoherent photon scattering rate. If the number of the scattered photons per atom per reflection is too high, the population of the chosen hyperfine ground sublevel is transferred to another one, for which the evanescent wave potential is too shallow or even attractive and reduces the number of reflected atoms. Incoherent photon 
scattering may be reduced by decreasing the penetration depth $d(\theta)$ and increasing the detuning $\Delta_{F F^{\prime}}$ (see Eq. (4)). On the other hand, this leads to lowering the mirror potential barrier. Successful dipole mirror preparation requires a kind of trade-off with two main parameters for fixed laser power: incidence angle $\theta$ and detuning $\Delta_{F F^{\prime}}$. Experimentally confirmed numerical calculations, involving atomprism interaction and photon scattering, show that the greatest number of reflected atoms occurs for $\theta$ exceeding the critical angle $\theta_{c}$ by roughly $2^{\circ}$ and $\Delta_{F F^{\prime}}$ of the order of $800 \mathrm{MHz}$.

The basic dipole mirror parameters were as follows: evanescent wave intensity $I_{0}=(90 \div 180) \times 10^{3} \mathrm{~W} / \mathrm{m}^{2}$ (at $z=0$, TM polarization $), \theta=1.5 \div 2.0^{\circ}$ over $\theta_{\mathrm{c}}$. The mean evanescent wave spot radius was $540 \mu \mathrm{m}$ and the detuning $\Delta_{F F^{\prime}}$ ranged between 350 and $2000 \mathrm{MHz}$ in respect of $5^{2} S_{1 / 2} F=3 \rightarrow 5^{2} P_{1 / 2}$ $F^{\prime}=3$ or $5^{2} S_{1 / 2} F=2 \rightarrow 5^{2} P_{1 / 2} F^{\prime}=3$ transitions. The initial height $h$ of the atom cloud was $0.3 \div 3.0 \mathrm{~mm}$. The calculated average number of incoherent scattered photons from the evanescent wave was $0.3 \div 1.5$ per reflection per atom.

The experimental sequence starts with $15 \mathrm{~s}$ of MOT loading. Then the magnetic field is switched off and (optional) $5 \mathrm{~ms}$ optical pumping stage is applied, during which in the absence of repumping light the atoms are transferred to the $F=2$ ground sublevel. In the next step all the beams going to the vacuum chamber are blocked by means of electromechanical shutters (with the switching time of the order of $0.5 \mathrm{~ms}$ ) and atoms fall under gravity towards the prism. After variable time of 15 to $70 \mathrm{~ms}$, cooling and repumping beams are switched on and fluorescent images of falling or reflected atoms are taken with the cooled CCD (Alta Apogee U32) camera with the $2184 \times 1472$ pixels array. The optical resolution of the imaging system was 27 pixels $/ \mathrm{mm}$ (with $2 \times 2$ hardware binning) and the integration time was 0.1 to $2 \mathrm{~ms}$. With this technique atoms both in the ground sublevels $F=2$ and $F=3$ were detected. The final image of the cloud resulted from the subtraction of two pictures — with and without atoms prepared in the MOT. This procedure allowed for background laser and laboratory light contribution suppression. For quantitative measurements the two-dimensional Gaussian profiles were fitted to the cloud images.

\section{Results}

In Fig. 3 a typical time sequence of falling and reflected atoms is presented. In the first image the atoms trapped in the MOT are shown. After releasing from the trap, the cold atomic cloud spreads ballistically and falls towards the prism. The width of the white rectangle in the first image corresponds to the Gaussian $\left(\mathrm{e}^{-2}\right)$ diameter of the evanescent wave spot on the prism. The calculated effective mirror surface is $\pi w_{\text {eff }}^{2}$ with $w_{\text {eff }}=380 \mu \mathrm{m}$. After about $23 \mathrm{~ms}$ the center of the cloud reaches the surface (image 3 ). In the images 4 to 8 the elastically reflected cloud is seen and in the images 7 and 8 the cloud is falling back to the prism. The two stationary spots on the left and right hand side of the images are the mounting screw heads reflections. 

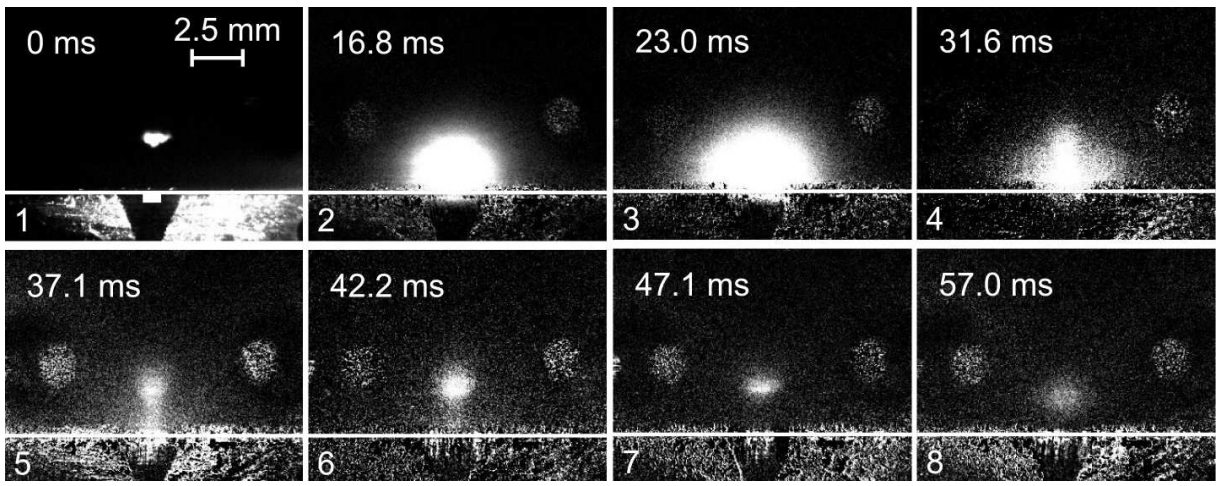

Fig. 3. Exemplary time sequence of bouncing atoms (see text). Evanescent wave detuning was $\Delta_{23}=2 \pi 900 \mathrm{MHz}$ in respect of $F=2 \rightarrow F^{\prime}=3$ transition, and initial MOT height was $h=2.5 \mathrm{~mm}$. The position of the prism surface is shown by white horizontal line.

The dipole mirror configuration used to present elastic bounces (see Fig. 3) allows also for the inelastic atom reflections. Let us assume that the atom scatters a photon close to the classical turning point. If the atom is transferred to another ground state (spontaneous Raman transition), for which the repulsive dipole potential is shallower, then its kinetic energy after the reflection is smaller than the initial one, as depicted in Fig. 4b. This process is called the Sisyphus cooling $[6,18,8]$. Its probability equals $P_{F m}\left(1-q_{\mathrm{hfs}}\right)$ for $P_{F m} \ll 1$, where $q_{\mathrm{hfs}}$ is the probability that the atom will remain in the same ground sublevel after photon scattering [18]. The probability $q_{\mathrm{hfs}}$ is close to 0.5 for $D_{1}$ line in alkali atoms. In Fig. 4a the elastically and inelastically reflected atoms are shown. The picture was obtained by subtraction of two images: with and without evanescent wave presence. It allowed for the suppression of the falling atoms contribution to the image. The vertical spreading of the atomic velocities (characteristic atomic column) results from the fact that the Sisyphus cooling efficiency depends on the place on the potential curve where the atom scatters the photon. The expected value of the energy reduction factor in the vertical direction is 0.47 here [18].

For quantitative results, the relative number of falling and reflected atoms was measured for different dipole mirror parameters. The evanescent wave detunings were chosen such that either atoms in the $F=2$ or $F=3$ state could be reflected. Several fluorescent images for the same dipole mirror parameters could be averaged thanks to a good reproducibility of the experimental sequences and atom cloud parameters. The mirror reflection coefficient was measured as a ratio between falling and reflected atom number and compared with the calculated one. The predicted reflection coefficient takes into account the falling cloud spatial distribution, the effective mirror surface and the incoherent photon scattering (see Sect. 2). The above procedure led to the conclusion that the losses in the re- 

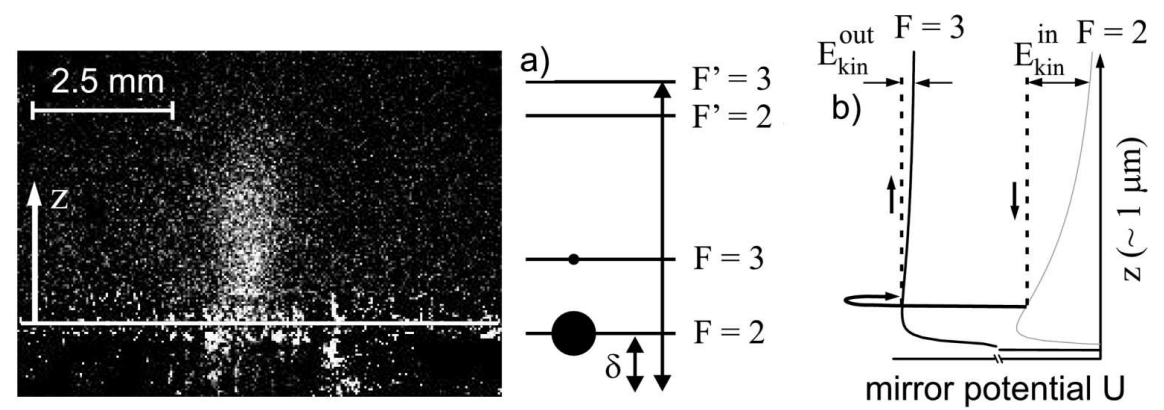

Fig. 4. (a) Atoms reflected elastically and inelastically, $29 \mathrm{~ms}$ after bounce. Evanescent wave detuning was $\Delta_{23}=2 \pi 900 \mathrm{MHz}$, initial MOT height was $h=2.5 \mathrm{~mm}$. Simple scheme of atom populations is shown (black circles); (b) basic explanation of the inelastic reflection (see text).

flected atoms, induced by scattered light on the prism surface imperfections, equal $25 \pm 5 \%$. The collisions with the background gas cause losses of the order of $12 \%$ of the reflected atoms for about $20 \mathrm{~ms}$ after reflection. The measured mirror reflection coefficient for typical experimental parameters is of the order of $1 \%$. This result follows the fact that the effective mirror surface is much smaller than the falling cloud cross-section. Additionally, it was calculated that when the optical pumping stage is not applied after the MOT phase, $70 \%$ of falling atoms are in the ground sublevel $F=3$.

\section{Conclusions and outlook}

Optical dipole mirror is a perfect tool in the field of atom-surface interaction studies as well as manipulation of atoms close to the surfaces. The presented setup is relatively cheap, simple, and flexible. It allows for observation of both elastically and inelastically reflected atoms and easy regulation of the initial height of the MOT cloud in the range of 0.3 to $8.0 \mathrm{~mm}$. The potential shape may be modified thanks to a wide range of accessible incident angles $\left(0-6^{\circ}\right.$ over $\left.\theta_{\mathrm{c}}\right)$. Simple fluorescence imaging is sufficient for quantitative measurements.

The reflection coefficient of the mirror was measured and a comparison with the calculations led to the estimation of the influence of the surface-scattered photons on the mirror performance.

The mirror gives reproducible results in terms of reflected atoms position and velocity distributions. Thus, several atom clouds images could be averaged for quantitative measurements.

Incoherent photon scattering will be further reduced below the level of 0.2 photons per bounce by increasing the evanescent wave detuning $\Delta_{F F^{\prime}}$ to $2 \pi 3.6 \mathrm{GHz}$. The mirror barrier will be maintained at the level given previously for $\Delta_{F F^{\prime}}=2 \pi 900 \mathrm{MHz}$ by doubling the evanescent wave intensity along with switching from $D_{1}$ to $D_{2}$ line. 
The setup will be used to investigate the evanescent wave radiation pressure acting on reflecting atoms. In particular, the incoherent photon scattering close to resonance will be measured for relatively weak additional evanescent wave, extending previous far-off-resonance measurements [21]. Providing that the on-surface intensity of this auxiliary evanescent wave lies in the range of 0.1 to $100 I_{\text {sat }}\left(I_{\text {sat }}\right.$ being the saturation intensity), the average number of scattered photons per bounce is 0.4 to 117 respectively, for dipole mirror parameters similar to the ones described in previous sections. With the $\mathrm{Rb}$ recoil velocity of the order of $6 \mathrm{~mm} / \mathrm{s}$, the momentum acquired by the reflected atoms will be measurable within resolution of our imaging system. The photon scattering will be investigated in dependence of the evanescent wave polarization state allowing for complementary studies to those presented in [22-24].

Improved stability and accuracy of the dipole mirror parameters will allow for precise probing of the QED corrections to the van der Waals potential [25, 26]. The important issue is to lower the impact velocity of falling atoms in comparison to the work [9]. This might be achieved by lowering the initial height of the MOT cloud to about $0.5 \mathrm{~mm}$ above the prism surface, which is easily accessible in our setup. These measurements require also lowering of the velocity dispersion of the falling atoms to the range of $10 \mu \mathrm{K}$. This will be attained by applying conventional molasses cooling stage. A kind of interferometric approach to the atom-wall interaction measurements, proposed in Ref. [27], also seems to be achievable, however certain extensions in the setup would be necessary.

\section{Acknowledgments}

Financial support by the State Committee for Scientific Research (Poland), grant No. 1PO3B09228, is greatly acknowledged.

\section{References}

[1] J. Dalibard, C. Cohen-Tannoudji, J. Opt. Soc. Am. B 2, 1707 (1985).

[2] M. Born, E. Wolf, Principles of Optics, 5th ed., Pergamon Press, New York 1975.

[3] R. Cook, R. Hill, Opt. Commun. 43, 258 (1982).

[4] V. Balykin, V. Letokhov, Y. Ovchinnikov, A. Sidorov, JETP Lett. 45, 353 (1987).

[5] M. Kasevich, D. Weiss, S. Chu, Opt. Lett. 15, 607 (1990).

[6] Y. Ovchinnikov, J. Söding, R. Grimm, JETP Lett. 61, 10 (1995).

[7] Y. Ovchinnikov, D. Laryushin, V. Balykin, V. Letokhov, JETP Lett. 62, 113 (1995).

[8] P. Desbiolles, M. Arndt, P. Szriftgiser, J. Dalibard, Phys. Rev. A 54, 4292 (1996).

[9] A. Landragin, J.-Y. Courtois, G. Labeyrie, N. Vansteenkiste, C. Westbrook, A. Aspect, Phys. Rev. Lett. 77, 1464 (1996).

[10] A. Steane, P. Szriftgiser, P. Desbiolles, J. Dalibard, Phys. Rev. Lett. 74, 4972 (1995). 
[11] M. Arndt, P. Szriftgiser, J. Dalibard, Phys. Rev. A 53, 3369 (1996).

[12] P. Szriftgiser, D. Guéry-Odelin, M. Arndt, J. Dalibard, Phys. Rev. Lett. 77, 4 (1996).

[13] D. Rychtarik, B. Engeser, H.-C. Nägerl, R. Grimm, Phys. Rev. Lett. 92, 173003 (2004).

[14] R. Folman, P. Krüger, J. Schmiedmayer, J. Denschlag, C. Henkel, Adv. At. Mol. Opt. Phys. 48, 263 (2002).

[15] C.W.C. Henkel, J. Schmiedmayer, Eur. Phys. J. D 35, 1 (2005).

[16] J. Dowling, J. Gea-Banacloche, Adv. At. Mol. Opt. Phys. 37, 1 (1996).

[17] R. Grimm, M. Weidemüller, Y. Ovchinnikov, Adv. At. Mol. Opt. Phys. 42, 95 (2000).

[18] J. Söding, R. Grimm, Y. Ovchinnikov, Opt. Commun. 119, 652 (1995).

[19] J.-Y. Courtois, J.-M. Courty, J.C. Mertz, Phys. Rev. A 53, 1862 (1996).

[20] K. Kiersnowski, Ph.D. Thesis, Jagiellonian University, Cracow 2007.

[21] D. Voigt, B.T. Wolschrijn, R. Jansen, N. Bhattacharya, R.J.C. Spreeuw, H. van Linden van den Heuvell, Phys. Rev. A 61, 063412 (2000).

[22] T. Kawalec, M. Kasprowicz, L. Józefowski, T. Dohnalik, Acta Phys. Pol. A 105, 349 (2004).

[23] L. Józefowski, J. Fiutowski, T. Kawalec, H.-G. Rubahn, J. Opt. Soc. Am. B 24, 624 (2007).

[24] T. Kawalec, L. Józefowski, M. Kasprowicz, T. Dohnalik, Opt. Commun. 274, 341 (2007).

[25] H. Casimir, D. Polder, Phys. Rev. 73, 360 (1948).

[26] S. Kallush, B. Segev, R. Côté, Eur. Phys. J. D 35, 3 (2005).

[27] R. Marani, L. Cognet, V. Savalli, N. Westbrook, C. Westbrook, A. Aspect, Phys. Rev. A 61, 053402 (2000). 